\title{
PRODUTIVIDADE DE CULTIVARES DE ALHO NA REGIÃO PAULISTA DE TIETÊ $\left({ }^{1}\right)$
}

\author{
PAULO ESPÍNDOLA TRANI $\left(2^{*}\right)$ DULCINÉIA ELIZABETE FOLTRAN $\left({ }^{3}\right)$; MÔNICA SARTORI \\ DE CAMARGO $\left({ }^{4}\right)$; SEBASTIÃO WILSON TIVELLI $\left({ }^{2}\right)$; FRANCISCO ANTONIO PASSOS $\left({ }^{2}\right)$
}

\begin{abstract}
RESUMO
O custo elevado das sementes (bulbilhos) de alho nobre e a vernalização podem aumentar a produtividade da cultura, mas dificultam seu cultivo por pequenos produtores, responsáveis pela maior parte da produção no Brasil. Assim, estudos de seleção de sementes de menor custo como os alhos comuns, feitos regionalmente, e de comparação com alho vernalizado quanto à produtividade, são fundamentais. O objetivo deste trabalho foi a avaliação da produtividade de quatro cultivares de alho comum não vernalizado (Assaí, Gigante de Curitibanos, Santa Catarina Branco e Santa Catarina Roxo), em comparação com alho chinês vernalizado Jonas (armazenamento dos bulbos a $4{ }^{\circ} \mathrm{C}$ por 40 dias). O delineamento experimental foi em blocos casualizados com cinco repetições. O experimento foi realizado em área experimental da APTA-UPD de Tietê (SP), de março a setembro de 2005. A variedade mais produtiva foi Jonas, sem diferir de Santa Catarina Roxo, Santa Catarina Branco e Assaí 3702, com 8,4; 7,6; 7,4 e 6,9 t ha-1 respectivamente e também com as maiores massas médias de bulbos $(30,5 ; 27,0 ; 26,3$ e 24,6g respectivamente). Quanto ao diâmetro do bulbo, os alhos comuns, exceto Gigante de Curitibanos, produziram bulbos tamanho $4(36-45 \mathrm{~mm})$. Para produtividade e massa do bulbo, a cultivar Gigante de Curitibanos foi inferior a Jonas.
\end{abstract}

Palavras-chave: Allium sativum L., avaliação regional, rendimento.

\section{ABSTRACT \\ GARLIC CULTIVARS YIELD IN TIETÊ REGION, SÃO PAULO STATE, BRAZIL}

The high cost of garlic seed and the refrigeration process may increase yield, but it is a barrier for small farmers who are responsible for the large Brazilian production. Therefore, research seeking low cost garlic seed, regionally made, and competition studies for yield between refrigerated and no refrigerated cultivars are fundamental. This work aimed to evaluate yield and bulb quality of four no refrigerated garlic varieties (Assaí 3702, Gigante de Curitibanos, Santa Catarina Branco and Santa Catarina Roxo) and the refrigerated type Jonas (storage at $4^{\circ} \mathrm{C}$ for 40 days). The field trial was carried out during March-September 2005, in Tietê, São Paulo State, Brazil, in a randomized block design with five replications. Jonas, without differ from Santa Catarina Roxo, Santa Catarina Branco, and Assaí 3702 produced higher yields $\left(8.4 ; 7.6 ; 7.4\right.$ and 6.9 t.ha $^{-1}$ respectively) and greater bulb weights $(30.5 ; 27.0 ; 26.3$ and $24.6 \mathrm{~g}$ respectively). For bulb diameter these four varieties were graded as type 4 (36-45mm), Gigante de Curitibanos was lower than Jonas concerning to yield and bulb mass.

Key words: Allium sativum L., regional evaluation, productivity.

$\left({ }^{1}\right)$ Recebido para publicação em 26 de janeiro de 2006 e aceito em $1 .^{\circ}$ de abril de 2008.

$\left({ }^{2}\right)$ Centro Avançado de Pesquisa e Tecnologia do Agronegócio de Horticultura, Caixa Postal 28, 13012-970 Campinas (SP),

E-mail: petrani@iac.sp.gov.br (*) Autor correspondente; tivelli@apta.sp.gov.br; fapassos@iac.sp.gov.br

$\left({ }^{3}\right)$ APTA-UPD de Tietê, Caixa Postal 18, 18530-970 Tietê (SP); E-mail: dulcineia@apta.sp.gov.br

$\left({ }^{4}\right)$ Apta Regional Centro-Sul, Caixa Postal 28, 13400-970 Piracicaba (SP) de Piracicaba. E-mail: mscamargo@apta.sp.gov.br. 


\section{Introdução}

O Brasil é um dos países com maior consumo de alho no mundo (Oliveira et al., 2003), tendo produzido 85 mil toneladas de alho em $10 \mathrm{mil}$ hectares, com rendimento médio de $8,5 \mathrm{t} \mathrm{ha}^{-1}$. Essa produção é insuficiente para atender a demanda nacional, sendo considerado o segundo maior importador (CAmARgo Filho e Alves, 2005). No Estado de São Paulo, a produtividade média ainda é baixa $\left(6,4 \mathrm{t} \mathrm{ha}^{-1}\right)$, requerendo a ampliação do uso de tecnologias como sementes de melhor qualidade, vernalização, e irrigação, além de tratos culturais específicos e ampliação das pesquisas com essa cultura (Lisbão et al., 1993; TAVARES et al.,1998).

Com a expansão da área cultivada com alhos nobres e roxos, em substituição aos alhos comuns e brancos, nas décadas de 80 e 90, aumentou o custo de produção da cultura do alho especialmente o da semente (33\% do custo total), enquanto o custo da vernalização foi de 5\% (TRANI et al., 1997). O custo elevado das sementes dificulta o cultivo de alhos nobres por pequenos produtores, responsáveis pela maior parte da produção de alho no Brasil (TRANI et al., 2005; MAROUElli et al., 2002). Os trabalhos realizados com a cultura (LISBÃo et al.,1991; LISBÃo et al., 1993; TrAni et al., 2005) têm mostrado que a produtividade das cultivares de alho são variáveis, de acordo com o manejo da cultura e as condições climáticas. Para a seleção de materiais promissores,a cultura exige a realização de experimentos regionais.

As avaliações de variedades têm sido feitas nos últimos 20 anos (LISBÃo et al.,1991; LisBÃo et al., 1993; TRANi et al., 2005) na região paulista de Tietê, mostrando o bom desempenho de algumas cultivares de alhos comuns, mas são necessários estudos comparativos com alho vernalizado. Atualmente, a área de plantio no Estado de São Paulo do alho chinês Jonas vernalizado tem sido ampliada em relação a outros tipos vernalizados como Roxo Pérola de Caçador e Quitéria (BoIANI, 2005) $\left(^{5}\right)$. Assim, o objetivo deste trabalho foi a avaliação da produtividade de quatro cultivares de alhos comuns não vernalizadas em comparação com alho chinês vernalizado 'Jonas' comumente plantado no Estado de São Paulo.

$\left({ }^{5}\right)$ Ismael Boiani, produtor de alho em Iacanga (SP).

\section{Material e Métodos}

O experimento foi realizado em área da APTA Regional Centro-Sul/Unidade de Pesquisa e Desenvolvimento de Tietê, no período de março a setembro de 2005, sendo o delineamento experimental em blocos casualizados com cinco repetições. Os tratamentos consistiram de quatro cultivares de alho do tipo comum (Assaí 3702, Gigante de Curitibanos, Santa Catarina Branco e Santa Catarina Roxo) e um tipo nobre e vernalizado (Jonas).

As cultivares Assaí 3702, Gigante de Curitibanos, Santa Catarina Branco e Santa Catarina Roxo, foram plantadas em 28/3/2005; a cultivar Jonas (após 40 dias de vernalização em câmara fria a $4{ }^{\circ} \mathrm{C}$ ) foi plantada em 4/5/2005.

Primeiramente, realizou-se a seleção dos bulbilhos com massa de 2,0 a 2,5 g em peneira número 2. O plantio do alho foi feito em parcelas de $1 \mathrm{~m} \times 2 \mathrm{~m}$, com quatro linhas de plantio longitudinais aos canteiros, os quais foram cobertos com grama batatais triturada e seca. O espaçamento foi de 0,25 m entre linhas de plantas e 0,10 m entre plantas na linha. A área útil da parcela consistiu das duas linhas centrais, totalizando $1 \mathrm{~m}^{2}$. Cada linha foi plantada com 20 bulbilhos, no total de 80 bulbilhos por parcela. Durante o decorrer do experimento, foi feito o controle manual de plantas daninhas, semanalmente, e também realizadas irrigações uniformes com uso de regador de crivos, a cada 2 dias na fase inicial (30 dias iniciais) e a cada três durante o restante do ciclo, suspendendo a irrigação 15 dias antes da colheita. As lâminas de água aplicadas basearam-se na exigência da cultura e na textura do solo. A calagem e adubação foram baseadas na recomendação para a cultura para o Estado de São Paulo (Trani et al., 1997).

Todas as variedades foram colhidas em $1 . \%$ $9 / 2005$. Foram avaliadas a produtividade $\left(\mathrm{t} \mathrm{ha}^{-1}\right)$, a massa média do bulbo (g) e o diâmetro médio do bulbo $(\mathrm{mm})$. A produtividade foi calculada através de método adotado por TRANI et al. (2005), considerandose como área útil $70 \%$ da área total do terreno. A massa média e o diâmetro médio do bulbo foram obtidos por meio da média aritmética de 20 bulbos por parcela.

Os dados foram submetidos ao teste de Hartley (5\% P), verificando-se não haver evidências de heterogeneidade de variâncias, dispensando o uso de transformações. Os dados originais foram submetidos à análise de variância e as médias, comparadas pelo teste de Tukey a $5 \%$ de significância. 


\section{Resultados e Discussão}

$\mathrm{Na}$ produtividade e na massa média dos bulbos observaram-se diferenças significativas entre as cultivares, ao contrário do ocorrido para o diâmetro médio do bulbo. $\mathrm{O}$ alho chinês Jonas (vernalizado) foi o mais produtivo com $8,4 \mathrm{t} \mathrm{ha}^{-1}$, sem diferir estatisticamente em relação às cultivares Santa Catarina Roxo, Santa Catarina Branco e Assaí 3702, que alcançaram produtividade de, respectivamente, 7,$6 ; 7,4$ e $6,9 \mathrm{t} \mathrm{ha}^{-1}$ (Tabela 1). Essas produtividades são semelhantes à média nacional $\left(8,6 \mathrm{t} \mathrm{ha}^{-1}\right)$ em 2004 (FAO, 2005) e à do Estado de São Paulo (4,0 a 8,0 t ha- $\left.{ }^{-1}\right)$ em anos anteriores (LisBão et al. 1993; TAVARES et al.,1998). Por outro lado, a produtividade da cultivar Gigante de Curitibanos, com 5,8 $\mathrm{t} \mathrm{ha}^{-1}$, foi significativamente inferior à produtividade do alho 'Jonas', sem diferir das demais variedades cultivadas (Tabela 1).

Tabela 1. Produtividade, massa média do bulbo e diâmetro do bulbo de variedades cultivadas de alho na fazenda experimental da APTA Regional/UPD de Tietê (SP), 2005

\begin{tabular}{lccc}
\hline Cultivar & Produtividade & Massa do bulbo $\left(^{1}\right)$ & Diâmetro do bulbo $\left(^{1}\right)$ \\
\hline & $\mathrm{t} \mathrm{ha}{ }^{-1}$ & $\mathrm{~g}$ & $\mathrm{~mm}$ \\
Jonas (vernalizado) & $8,44 \mathrm{a}$ & $30,46 \mathrm{a}$ & $37,45 \mathrm{a}$ \\
Santa Catarina Roxo & $7,56 \mathrm{ab}$ & $27,00 \mathrm{ab}$ & $36,86 \mathrm{a}$ \\
Santa Catarina Branco & $7,36 \mathrm{ab}$ & $26,30 \mathrm{ab}$ & $36,89 \mathrm{a}$ \\
Assaí 3702 & $6,89 \mathrm{ab}$ & $24,60 \mathrm{ab}$ & $36,28 \mathrm{a}$ \\
Gigante de Curitibanos & $5,84 \mathrm{~b}$ & $20,80 \mathrm{~b}$ & $34,69 \mathrm{a}$ \\
\hline C.V. $(\%)$ & 12,50 & 12,57 & 4,99 \\
F $\left(^{2}\right)$ & $3,03^{*}$ & $3,15^{*}$ & $0,96 \mathrm{~ns}$ \\
DMS $\left({ }^{3}\right)$ & 1,74 & 6,30 & 3,53
\end{tabular}

( $\left.{ }^{1}\right)$ Com base na média aritmética de 20 bulbos por parcela

$\left({ }^{2}\right)$ Valor de F relativo à análise de variância: efeito significativo $\left({ }^{*}\right)$ e não significativo (ns) de variedades cultivadas a 5 \%

$\left({ }^{3}\right)$ Diferença mínima significativa pelo Teste de Tukey a $5 \%$.

As produtividades das cultivares de alho neste ensaio foram semelhantes àquelas obtidas na mesma região por LisB̃̃o et al. (1991), quando corrigidas conforme proposto por TRANI et al. (2005), realizado com outras variedades. Em trabalho de LISBÃo et al. (1991), a cultivar Lavínia produziu 8,4 t $\mathrm{ha}^{-1}$. No experimento de TrAni et al. (2005), em Tietê, 'Gigante de Curitibanos' $\left(10,9 \mathrm{t} \mathrm{ha}^{-1}\right)$ foi superior à 'Santa Catarina Branco' (5,6 t ha-1) e 'Assaí 3702' (4,1 $\left.\mathrm{t} \mathrm{ha}^{-1}\right)$, contrastando com os resultados constantes deste experimento. Esse comportamento diferenciado das cultivares de alho, provavelmente ocorreu devido a variações nas condições climáticas, sobretudo, temperatura e tratos culturais como irrigação e adubação que podem variar de um ano para outro. As temperaturas no início da formação de bulbos (7090 dias após o plantio) e na época de formação de bulbilhos (110 a 120 dias após o plantio) que precisam ser baixas $\left(<12{ }^{\circ} \mathrm{C}\right)$ variaram de 8,8 a $27^{\circ} \mathrm{C}$, em 2003 e de 7,6 a $28,6{ }^{\circ} \mathrm{C}$ em 2005, influenciando, provavelmente, a cultivar Gigante de Curitibanos de maneira mais intensa do que as demais.

O alho chinês Jonas produziu bulbos com maior massa média (30,5 g), em magnitude, o qual acabou sendo semelhante à massa média dos bulbos de 'Santa Catarina Roxo', 'Santa Catarina Branco' e 'Assaí 3702', que produziram bulbos com 27,0, 26,3 e 24,6 g respectivamente. Esses resultados são semelhantes aos obtidos para a produtividade, estando também relacionados ao tamanho e ao volume do bulbo. Por sua vez, 'Gigante de Curitibanos' com a menor massa média por bulbo $(20,8 \mathrm{~g})$, foi inferior à massa média dos bulbos de 'Jonas' e semelhante às demais variedades cultivadas.

Para as cultivares utilizadas neste estudo, embora não tenha ocorrido diferença estatística, foi observado tamanho 4 (36-45 mm), exceto em 'Gigante de Curitibanos', que se enquadrou no tamanho 3 (26$35 \mathrm{~mm}$ ), bem inferior aos 44,6 mm obtido em 2003 por TRANi et al. (2005). Esse fato pode ter ocorrido devido à maior sensibilidade às temperaturas durante a fase de formação de bulbilhos, que não foi favorável neste experimento. A uniformidade do bulbo quanto à sua forma tem implicações na comercialização. Verificou-se neste trabalho que o alho chinês Jonas tem os bulbos mais esféricos que as demais cultivares de alho não vernalizadas, o que contribui para melhor cotação de preços. Para os alhos comuns, essa característica não é tão importante como a produtividade, pois é feita sua industrialização anteriormente à comercialização. Algumas características morfológicas (cor e forma) dos bulbos e bulbilhos dos alhos 'Jonas', 'Santa Catarina Roxo' e 'Assaî' são apresentadas nas Figuras 1 e 2 . 


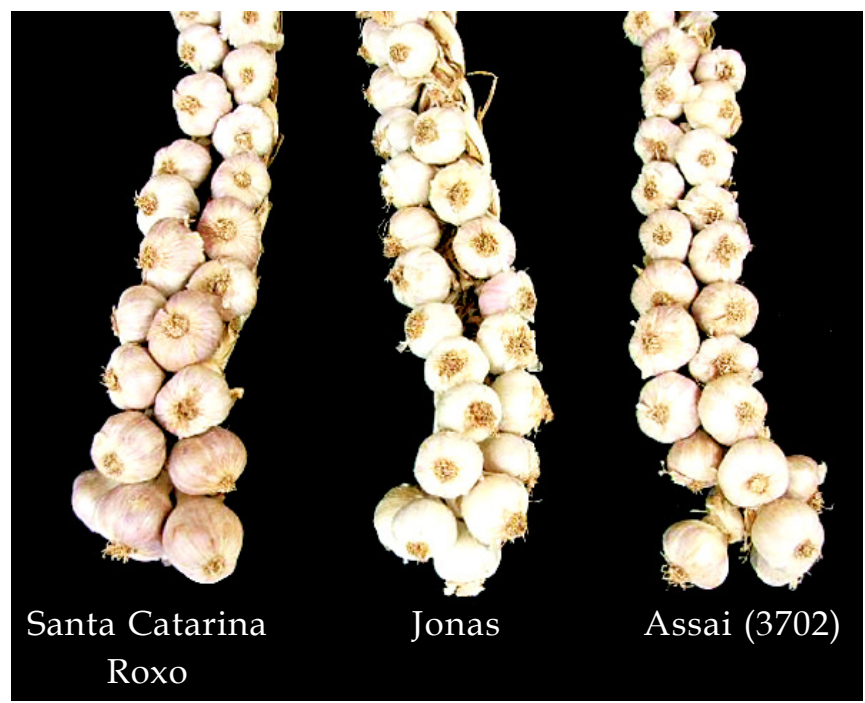

Figura 1. Aspecto dos bulbos de três cultivares de alho avaliadas em Tietê (SP), em 2005.

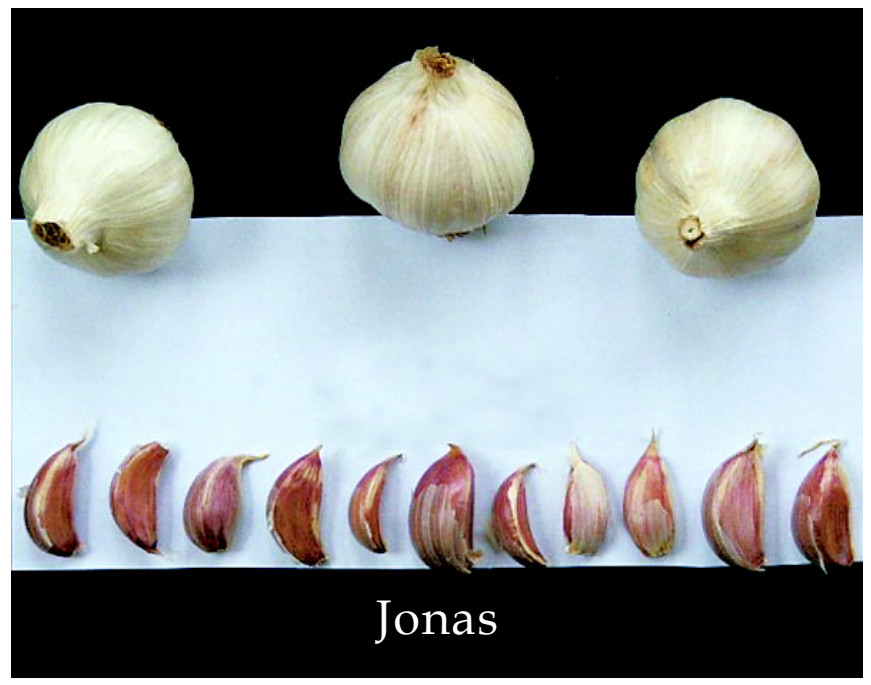

Figura 2. Aspecto do bulbo e bulbilhos do alho vernalizado 'Jonas', em Tietê (SP), em 2005.

\section{Conclusões}

1. Considerando-se as variáveis analisadas, verificou-se superioridade do alho Jonas vernalizado, sobre a cultivar Gigante de Curitibanos quanto à produtividade e massa do bulbo.

2. As cultivares de alho não vernalizadas Santa Catarina Roxo, Santa Catarina Branco e Assaí não diferiram estatísticamente de Jonas, constituindose em opção de cultivo aos produtores de alho da região de Tietê que não tem acesso ao processo de vernalização.

\section{Agradecimentos}

Os autores agradecem ao produtor Ismael Boiani, de Iacanga (SP), pela doação dos bulbilhos de alho da cultivar Jonas e pelo incentivo à realização desse trabalho.

\section{Referências}

CAMARGO FILHO, W. P., ALVES, H. S. Mercado de alho no Mercosul: produção, estacionalidade e consolidação do mercado. Informações Econômicas, São Paulo, v. 35, n. 7, p. 18-27, Jul. 2005.

FAO - FOOD AND AGRICULTURE ORGANIZATION OF THE UNITED NATIONS FAOSTAT: Agricultural Production Garlic (last updated 14/07/2005). Disponível em: <http:// $\mathrm{f}$ a o s t a t.f a o o r g / f a o s t a t / s e r v le t / XteServlet3?Areas $=\% 3 E 872 \&$ Items $=406 \&$ Elements $=51 \&$ Years $=$ $2004 \&$ Format $=$ Table $\&$ Xaxis $=$ Years $\&$ Yaxis $=$ Countries $\&$ Aggregate $=\&$ Calculate $=\&$ Domain $=$ SUA\&ItemTypes $=$ Production.Crops. Primary\&language $=E N>$. Acesso em: 14 dez. 2005.

LISBÃO, R.S.; FORNASIER, J.B.; GROPPO, G.A.; NEVES, J.P.S.; BARBOSA, L.A.V.; INADA, I.; RODRÍGUEZ, A.; VERAGUAS, S.; FERNÁNDEZ, S.C.S. Avaliação de cultivares de alho. Campinas: Coordenadoria de Assistência Técnica Integral/Programa de Pesquisa e Adaptação de Tecnologias Regionais,.1991. p. 22.

LISBÃO, R.S.; SIQUEIRA, W.J.; FORNASIER, J.B.; TRANI, P.E. Alho. In: FURLANI, A.M.C.; VIEGAS, G.P., Ed. O melhoramento de plantas no Instituto Agronômico. Campinas: Instituto Agronômico, 1993. p.222-253.

MAROUELLI, W.A.; SILVA, W.L.C.; CARRIJO, O.A.; SILVA, H.R. Produção e qualidade de alho sob regimes de água no solo e doses de nitrogênio. Horticultura Brasileira, Brasília, v. 20, n.2, p.191-194, 2002.

OLIVEIRA, C.M.;SOUZA, R.J.;MOTA, J.H.; YURI, J.E.; RESENDE, G.M. Determinação do ponto de colheita na produção de alho. Horticultura Brasileira, Brasília, v. 21, n. 3, p. 506-509, 2003.

SOUZA, A.T. Aspectos econômicos da cultura do alho. Disponível em: <www.icepa.com.br/agroindicadores/ opiniao/analise_alho.htm>. Acesso em 25 nov. 2005.

TAVARES, M.; TRANI, P. E.; SIQUEIRA, W. J. Alho. In: FAHL, J. I.; CAMARGO, M. B. P.; PIZZINATTO, M. A.; BETTI, J. A.; MELO, A. M. T.; DEMARIA, I. C.; FURLANI, A. M. C. Instruções agrícolas para as principais culturas econômicas. 6a. ed. rev. atual. Campinas: Instituto Agronômico, 1998. p. 175-176. (Boletim IAC, 200)

TRANI, P.E.; TAVARES, M.; SIQUEIRA, W.J.; SANTOS, R.R.; BISÃO, L.G.; LISBÃO, R.S. Cultura do alho: recomendações para seu cultivo no Estado de São Paulo. Campinas: Instituto Agronômico, 1997. 39p. (Boletim Técnico, 170)

TRANI, P.E.; PASSOS, F.A.; FOLTRAN, D.E.; TIVELLI, S.W.; RIBEIRO, I.J.A. Avaliação dos acessos de alho pertencentes à coleção do Instituto Agronômico de Campinas. Horticultura Brasileira, Brasília, v. 23, n. 4, p. 935-939, Out-Dez. 2005. 